\title{
Differential association of HLA with three subtypes of type 1 diabetes: fulminant, slowly progressive and acute-onset
}

\author{
Y. Kawabata • H. Ikegami - T. Awata • A. Imagawa • \\ T. Maruyama - E. Kawasaki - S. Tanaka • A. Shimada • \\ H. Osawa $\cdot$ T. Kobayashi $\cdot$ T. Hanafusa $\cdot$ K. Tokunaga $\cdot$ \\ H. Makino $\cdot$ on behalf of the Committee on Type 1 \\ Diabetes, Japan Diabetes Society
}

Received: 6 June 2009 / Accepted: 26 August 2009 /Published online: 8 October 2009

(C) Springer-Verlag 2009

\begin{abstract}
Aim/hypothesis We sought to clarify similarities and differences in the contribution of HLA to genetic susceptibility to three subtypes of type 1 diabetes: acute-onset, fulminant and slowly progressive.
\end{abstract}

Electronic supplementary material The online version of this article (doi:10.1007/s00125-009-1539-9) contains a list of members of the Committee on Type 1 Diabetes, Japan Diabetes Society, which is available to authorised users.

Y. Kawabata $\cdot$ H. Ikegami $(\bowtie)$

Department of Endocrinology,

Metabolism and Diabetes,

Kinki University School of Medicine,

377-2 Ohno-higashi, Osaka-sayama,

Osaka 589-8511, Japan

e-mail: ikegami@med.kindai.ac.jp

T. Awata

Department of Endocrinology and Diabetes,

Saitama Medical University,

Saitama, Japan

A. Imagawa

Department of Metabolic Medicine,

Graduate School of Medicine, Osaka University,

Osaka, Japan

T. Maruyama

Department of Internal Medicine,

Saitama Social Insurance Hospital,

Saitama, Japan

E. Kawasaki

Department of Metabolism/Diabetes and Clinical Nutrition, Nagasaki University Hospital of Medicine and Dentistry,

Nagasaki, Japan
Methods We genotyped 545 Japanese patients with type 1 diabetes (338 acute-onset, 80 fulminant, 127 slowly progressive) and 396 control participants at $H L A-D R B 1,-D Q B 1,-A$, $-B$ and $-C$, and at 101 candidate single nucleotide polymorphisms (SNPs) in an $8.5 \mathrm{Mb}$ region of the extended HLA.

S. Tanaka $\cdot$ T. Kobayashi

Third Department of Internal Medicine,

Interdisciplinary Graduate School of Medicine and Engineering,

University of Yamanashi,

Yamanashi, Japan

A. Shimada

Department of Internal Medicine, Keio University School of Medicine, Tokyo, Japan

H. Osawa

Department of Molecular and Genetic Medicine,

Ehime University Graduate School of Medicine,

Ehime, Japan

T. Hanafusa

First Department of Internal Medicine, Osaka Medical College,

Osaka, Japan

K. Tokunaga

Department of Human Genetics, Graduate School of Medicine,

The University of Tokyo,

Tokyo, Japan

H. Makino

Takanoko Hospital Diabetes Center,

Ehime, Japan 
Results DRB1*0405-DQB1*0401, DRB1*0802-DQB1* 0302 and $D R B 1 * 0901-D Q B 1 * 0303$ were associated with acute-onset type 1 diabetes, with the $D R B 1^{*} 0405-D Q B 1 *$ $0401 / D R B 1 * 0802-D Q B 1 * 0302$ genotype achieving the highest odds ratio of 42.7. DRB1*1501-DQB1*0602 and $D R B 1 * 1502-D Q B 1 * 0601$ were negatively associated with acute-onset type 1 diabetes. A similar tendency was observed for slowly progressive type 1 diabetes. In contrast, only $D R B 1 * 0405-D Q B 1 * 0401$ was associated with fulminant type 1 diabetes, with the $D R B 1 * 0405-D Q B 1 * 0401 /$ $D R B 1 * 0405-D Q B 1 * 0401$ genotype showing the highest odds ratio of 11.2. $D R B 1 * 0802-D Q B 1 * 0302, D R B 1 * 0405$ $D Q B 1 * 0401 / D R B 1 * 0802-D Q B 1 * 0302$ and $D R B 1 * 1501$ $D Q B 1 * 0602$ were not associated with fulminant type 1 diabetes. The association of class I alleles and a panel of SNPs in an extended HLA region with fulminant type 1 diabetes was also different from that seen for the acuteonset and slowly progressive forms. The presence of both one and two susceptible haplotypes conferred susceptibility to slowly progressive type 1 diabetes, whereas the presence of two susceptible haplotypes was required to confer susceptibility to acute-onset and fulminant type 1 diabetes. Conclusions/interpretation These data suggest that HLA associations with fulminant type 1 diabetes are qualitatively different from those with other subtypes of type 1 diabetes, whereas the HLA contribution to slowly progressive type 1 diabetes is qualitatively similar to, but quantitatively different from, that in acute-onset type 1 diabetes.

Keywords Fulminant type 1 diabetes - Genetics · HLA · Single nucleotide polymorphism - Slowly progressive type 1 diabetes $\cdot$ Susceptibility Type 1 diabetes

Abbreviations
$\begin{array}{ll}\text { LADA } & \text { Latent autoimmune diabetes in adults } \\ \text { SNP } & \text { Single nucleotide polymorphism } \\ \text { UTR } & \text { Untranslated region }\end{array}$

\section{Introduction}

Type 1 diabetes is a clinically and aetiologically heterogeneous disorder. In addition to typical acute-onset type 1 diabetes, at least two subtypes have been described: fulminant and slowly progressive. Fulminant type 1 diabetes, which is characterised by an extremely acute onset of diabetes and absence of islet-related autoantibodies [1], accounts for up to $20 \%$ of type 1 diabetes in Japan [2] and $7 \%$ in Korea [3]. Slowly progressive type 1 diabetes, in contrast, is characterised by positivity for islet-related autoantibodies, but a long non-insulin-dependent stage, lasting usually for years, with gradual loss of beta cells leading ultimately to an insulin-dependent stage [4]. Although all three subtypes share the same clinical characteristic of insulin dependence in the final stage, the time course of beta cell destruction is markedly different, which may well be based on differences in the aetiology, including genetic susceptibility, among the three subtypes of the disease $[2,4]$.

The contribution of HLA, in particular class II DR and DQ genes, to susceptibility to autoimmune type 1 diabetes has been well described [5]. The HLA alleles contributing to fulminant type 1 diabetes appear to be different from those in autoimmune type 1 diabetes. In an initial report [1], high frequencies of class II HLA alleles known to provide resistance to type 1 diabetes were described. Subsequent studies with serological typing of class II HLA in patients recruited through a nationwide survey showed a higher frequency of DR4-DQ4 as well as DR2-DQ1 haplotypes in fulminant type 1 diabetes than in autoimmune type 1 diabetes [6]. Slowly progressive type 1 diabetes was also reported to be associated with class II HLA [4].

Despite the contribution of HLA to each subtype of type 1 diabetes, no extensive studies comparing HLA alleles and genotypes in the three subtypes of type 1 diabetes have been performed, probably because the low frequency of type 1 diabetes in Japan makes it difficult to collect substantial numbers of samples for all three subtypes at any given institute. To overcome this, the Committee on Type 1 Diabetes of the Japan Diabetes Society has been performing nationwide studies and collecting samples from patients with fulminant as well as the typical acute-onset and slowly progressive subtypes of type 1 diabetes. Taking advantage of these samples, we studied the associations of a panel of single nucleotide polymorphism (SNP) markers in the HLA region as well as class I and class II alleles of HLA with all three subtypes of type 1 diabetes. Our aim was to clarify the similarities and differences in the contribution of HLA to genetic susceptibility to the three subtypes.

\section{Methods}

Participants We studied 545 Japanese patients with type 1 diabetes (338 typical acute-onset, 80 fulminant and 127 slowly progressive type 1 diabetes) and 396 healthy control participants. Patients with fulminant type 1 diabetes were recruited through the Japan Diabetes Society as described previously [6]. Samples from patients with acute-onset and slowly progressive type 1 diabetes were collected from the hospitals of the committee members. The patients were ketosis-prone and positive for at least one of the isletrelated autoantibodies, i.e. GAD antibodies, islet cell antibodies, insulin autoantibodies and/or IA-2 antibodies. 
The duration of hyperglycaemic symptoms before the start of insulin therapy was less than 3 months for typical acuteonset type 1 diabetes and more than 12 months for slowly progressive type 1 diabetes. This study was approved by the appropriate ethics committees and informed consent was obtained from all participants.

Genotyping of class II and class I HLA Class II DRBI and $D Q B 1$ were genotyped by the PCR sequence-specific primer and PCR sequence-specific oligonucleotide methods (Invitrogen, Carlsbad, CA, USA). The most probable $D R B 1-D Q B 1$ haplotypes were deduced from known linkage disequilibria. Class I A, B and C alleles were genotyped by PCR sequence-specific oligonucleotide method (Wakunaga Pharmaceutical, Hiroshima, Japan).

Genotyping of SNPs in HLA region A total of 101 SNPs in 76 candidate genes in an $8.5 \mathrm{Mb}$ region of the HLA was selected. Selection of candidate genes and SNPs was based on the following criteria: candidate genes reported in the literature and identified on PubMed using the following keywords: type 1 diabetes, insulin-dependent diabetes mellitus, gene, human. In addition, genes related to immunological function and those expressed in the pancreas were also included. When multiple SNPs were identified in the same gene, SNPs were prioritised as follows: (1) SNPs reported to have a significant association with the disease; (2) SNPs in coding sequences; (3) SNPs in promoter, $5^{\prime}$ and $3^{\prime}$ untranslated regions; and (4) SNPs in introns. Within the same category, priority was given to SNPs uploaded in JSNP (http://snp.ims.u-tokyo.ac.jp/index. $\mathrm{html}$, accessed 4 March 2009) with minor allele frequencies $>0.05$ in Japanese.

Genotyping was performed by the PCR fluorescence correlation spectroscopy method at the SNP Typing Center in the Department of Human Genetics, Tokyo University, as reported previously [7]. Initially, 16 samples commonly used at the Typing Center for pilot screening for polymorphisms were typed, with only SNPs confirmed to show polymorphism in at least one sample being used for further typing. Nine SNPs not polymorphic in the initial screening and 24 SNPs that showed either non-specific amplification due to homologous pseudogenes or low calling rates were omitted. The remaining 68 SNPs were used for typing of all samples.

Statistical analysis Statistical analysis was performed with StatView 5.0 (SAS Institute, Cary, NC, USA). The significance of differences in the distribution of alleles was determined by the $\chi^{2}$ test or Fisher's exact probability test. $p$ values were corrected for the number of different alleles tested (denoted as $p_{\mathrm{c}}$ ). Statistical significance was defined as $p<0.05$.

\section{Results}

Class II alleles and haplotypes Association of class II HLA alleles and haplotypes with all three subtypes of type 1 diabetes was observed (Table 1, Electronic supplementary material [ESM] Tables 1, 2 and 3). However, a marked difference in the alleles and haplotypes associated with the disease was observed among the three subtypes. As reported previously [8-11], the $D R B 1 * 0405$ and *0901 alleles (ESM Table 1), $D Q B 1 * 0401$ and $* 0303$ alleles (ESM Table 2), and $D R B 1 * 0405-D Q B 1 * 0401$, DRB1*0802$D Q B 1 * 0302$ and $D R B 1 * 0901-D Q B 1 * 0303$ haplotypes (Table 1) were associated with acute-onset type 1 diabetes. The $D R B 1 * 1501$ and *1502 alleles (ESM Table 1), $D Q B 1 *$ 0601 and $D Q B 1^{*} 0602$ alleles (ESM Table 2), and $D R B 1^{*}$ 1501-DQB1*0602 and DRB1*1502-DQB1*0601 haplotypes (Table 1$)$ were negatively associated with acute-

Table $1 D R B 1-D Q B 1$ haplotypes associated positively (susceptible) or negatively (protective) with disease in patients with acute-onset, fulminant and slowly progressive type 1 diabetes, and in control participants

\begin{tabular}{|c|c|c|c|c|c|c|c|c|c|c|c|c|c|c|}
\hline \multirow[t]{2}{*}{$D R B 1-D Q B 1$} & \multicolumn{2}{|c|}{ Control $(n=792)$} & \multicolumn{2}{|c|}{ Acute $(n=676)$} & \multicolumn{2}{|c|}{ Fulminant $(n=160)$} & \multicolumn{2}{|c|}{ SP $(n=254)$} & \multicolumn{2}{|l|}{ A vs $\mathrm{C}$} & \multicolumn{2}{|l|}{ F vs $\mathrm{C}$} & \multicolumn{2}{|l|}{$\mathrm{SP}$ vs $\mathrm{C}$} \\
\hline & $n$ & $\%$ & $n$ & $\%$ & $n$ & $\%$ & $n$ & $\%$ & $p_{\mathrm{c}}$ value & OR & $p_{\mathrm{c}}$ value & OR & $p_{\mathrm{c}}$ value & OR \\
\hline \multicolumn{15}{|l|}{ Susceptible } \\
\hline$* 0405-* 0401$ & 96 & 12.1 & 205 & 30.3 & 51 & 31.9 & 65 & 25.6 & $2.6 \times 10^{-16}$ & 3.2 & $1.2 \times 10^{-7}$ & 3.4 & $4.5 \times 10^{-6}$ & 2.5 \\
\hline *0802-*0302 & 14 & 1.8 & 48 & 7.1 & 3 & 1.9 & 13 & 5.1 & $1.6 \times 10^{-5}$ & 4.3 & NS & 1.1 & NS & 3.0 \\
\hline *0901-*0303 & 117 & 14.8 & 209 & 30.9 & 38 & 23.8 & 62 & 24.4 & $3.8 \times 10^{-12}$ & 2.6 & NS & 1.8 & $7.8 \times 10^{-3}$ & 1.9 \\
\hline \multicolumn{15}{|l|}{ Protective } \\
\hline$* 1501-* 0602$ & 59 & 7.4 & 6 & 0.9 & 11 & 6.9 & 7 & 2.8 & $4.9 \times 10^{-8}$ & 0.11 & NS & 0.92 & NS & 0.35 \\
\hline$* 1502-* 0601$ & 80 & 10.1 & 21 & 3.1 & 8 & 5.0 & 4 & 1.6 & $4.6 \times 10^{-6}$ & 0.29 & NS & 0.47 & $2.7 \times 10^{-4}$ & 0.14 \\
\hline
\end{tabular}

For complete list of haplotypes including those not associated with the disease, see ESM Table 3

A, acute-onset; $\mathrm{C}$, control participants; $\mathrm{F}$, fulminant; $p_{\mathrm{c}}, p$ values corrected for number of different haplotypes tested; SP, slowly progressive 
onset type 1 diabetes. The alleles and haplotypes associated with slowly progressive type 1 diabetes were similar to those associated with acute-onset type 1 diabetes (Table 1, ESM Tables 1, 2 and 3). In contrast, the alleles and haplotypes associated with fulminant type 1 diabetes were different from those associated with other subtypes. The strong negative association of the $D R B 1 * 1501$ and $* 1502$ alleles, the $D Q B 1 * 0602$ alleles and the $D R B 1 * 1501$ $D Q B 1^{*} 0602$ and $D R B 1^{*} 1502-D Q B 1 * 0601$ haplotypes with acute-onset type 1 diabetes was not observed in fulminant type 1 diabetes. In fact, the frequency of a strongly protective haplotype, $D R B 1 * 1501-D Q B 1 * 0602$, was significantly higher in fulminant type 1 diabetes than in acuteonset type 1 diabetes $\left(6.9 \%\right.$ vs $\left.0.9 \%, p_{\mathrm{c}}=2.8 \times 10^{-5}\right)$ and was similar to that in control participants $(7.4 \%)$. The positive association of the $D R B 1 * 0802-D Q B 1 * 0302$ haplotype observed in acute-onset type 1 diabetes (OR 4.3, $p_{\mathrm{c}}=$ $1.6 \times 10^{-5}$ ) was not observed in fulminant type 1 diabetes (OR 1.1, NS) (Table 1).

Class II genotypes Homozygosity for DRB $1 * 0405$ $D Q B 1 * 0401$ was associated with all three subtypes of type 1 diabetes. The frequency, however, was much higher in fulminant type 1 diabetes $(12.5 \%$, OR 11.2$)$ than in the acute-onset (8.3\%, OR 7.1$)$ and slowly progressive $(7.1 \%$, OR 6.0) subtypes, although the difference between the groups was not statistically significant (Table 2). In contrast, the frequency of $D R B 1^{*} 0901-D Q B 1^{*} 0303$ homozygotes was higher in acute-onset $(15.7 \%)$ than in fulminant $(7.5 \%)$ and slowly progressive $(7.9 \%)$ type 1 diabetes (Table 2). The DR4/8 (DRB1*0405-DQB1*0401/DRB1* $0802-D Q B 1 * 0302)$ genotype was significantly associated with acute-onset (OR 42.7, $\left.p_{\mathrm{c}}=5.6 \times 10^{-10}\right)$ and slowly progressive (OR 16.2, $p_{\mathrm{c}}=0.03$ ) type 1 diabetes, but not with fulminant type 1 diabetes (Table 1); the frequency in fulminant type 1 diabetes was lower than in acute-onset type 1 diabetes $(9.8 \%$ vs $1.3 \%, p=0.01)$.

On the basis of their association with acute-onset type 1 diabetes, haplotypes were classified as susceptible (S) (haplotypes $D R B 1 * 0405-D Q B 1 * 0401, D R B 1 * 0802$ $D Q B 1 * 0302$ and $D R B 1 * 0901-D Q B 1 * 0303)$, protective $(\mathrm{P})$ (haplotypes $D R B 1 * 1501-D Q B 1 * 0602$ and $D R B 1 * 1502$ $D Q B 1^{*} 0601$ ) or neutral (N) (haplotypes other than susceptible and protective haplotypes). Within this classification, the presence of two susceptible haplotypes $(\mathrm{S} / \mathrm{S})$ was associated with acute-onset (OR 10.0, $\left.p_{\mathrm{c}}=2.6 \times 10^{-35}\right)$ and fulminant type 1 diabetes (OR 5.7, $p_{\mathrm{c}}=4.7 \times 10^{-8}$ ), but the presence of one susceptible haplotype $(\mathrm{S} / \mathrm{N}, \mathrm{S} / \mathrm{P})$ had no effect on susceptibility to the disease (Table 3). In contrast, the presence of both two $(\mathrm{S} / \mathrm{S})$ and of one $(\mathrm{S} / \mathrm{N})$ susceptible haplotype was associated with slowly progressive type 1 diabetes (OR 4.1, $p_{\mathrm{c}}=8.5 \times 10^{-8}$; OR 2.4, $p_{\mathrm{c}}=1.1 \times 10^{-4}$, respectively) (Table 3 ).

Protective haplotypes provided strong protection against acute-onset (OR $\left.0.17, p_{\mathrm{c}}=2.0 \times 10^{-17}\right)$ and slowly progressive type 1 diabetes (OR $0.19, p_{\mathrm{c}}=3.4 \times 10^{-7}$ ), but no such effect was observed for fulminant type 1 diabetes (OR 0.58 , NS) (Table 3). Thus, the susceptibility and protection provided by HLA haplotypes differed among the three subtypes of type 1 diabetes. In acute-onset type 1 diabetes $\mathrm{S} / \mathrm{S}$ provided susceptibility and $\mathrm{P}$ provided protection, while $\mathrm{S} / \mathrm{S}$ provided susceptibility but with no protective haplotypes in fulminant type 1 diabetes; in the slowly progressive subtype, both $\mathrm{S} / \mathrm{S}$ and $\mathrm{S} / \mathrm{N}$ provided susceptibility and $\mathrm{P}$ provided protection.

Class I HLA alleles and genotypes As seen in ESM Tables 4, 5, and 6, the frequency of $B^{*} 5401$ was

Table $2 D R B 1-D Q B 1$ genotypes in patients with acute-onset, fulminant and slowly progressive type 1 diabetes, and in control participants

\begin{tabular}{|c|c|c|c|c|c|c|c|c|c|c|c|c|c|c|}
\hline \multirow[t]{2}{*}{$D R B 1-D Q B 1$} & \multicolumn{2}{|c|}{ Control $(n=396)$} & \multicolumn{2}{|c|}{ Acute $(n=338)$} & \multicolumn{2}{|c|}{ Fulminant $(n=80)$} & \multicolumn{2}{|c|}{$\mathrm{SP}(n=127)$} & \multicolumn{2}{|l|}{ A vs $\mathrm{C}$} & \multicolumn{2}{|l|}{$\mathrm{F}$ vs $\mathrm{C}$} & \multicolumn{2}{|l|}{ SP vs C } \\
\hline & $n$ & $\%$ & $n$ & $\%$ & $n$ & $\%$ & $n$ & $\%$ & $p_{\mathrm{c}}$ value & OR & $p_{\mathrm{c}}$ value & OR & $p_{\mathrm{c}}$ value & OR \\
\hline$D R 4 / 4$ & 5 & 1.3 & 28 & 8.3 & 10 & 12.5 & 9 & 7.1 & $4.3 \times 10^{-5}$ & 7.1 & $1.4 \times 10^{-6}$ & 11.2 & 0.014 & 6.0 \\
\hline DR9/9 & 12 & 3.0 & 53 & 15.7 & 6 & 7.5 & 10 & 7.9 & $9.8 \times 10^{-9}$ & 6.0 & NS & 2.6 & NS & 2.7 \\
\hline$D R 4 / 9$ & 15 & 3.8 & 46 & 13.6 & 11 & 13.8 & 12 & 9.4 & $1.4 \times 10^{-5}$ & 4.0 & 0.013 & 4.1 & NS & 2.7 \\
\hline$D R 4 / 8$ & 1 & 0.3 & 33 & 9.8 & 1 & 1.3 & 5 & 3.9 & $5.6 \times 10^{-10}$ & 42.7 & NS & 5.0 & 0.034 & 16.2 \\
\hline DR8/9 & 3 & 0.8 & 9 & 2.7 & 1 & 1.3 & 1 & 0.8 & NS & 3.6 & NS & 1.7 & NS & 1.0 \\
\hline$D R 4 / X$ & 70 & 17.7 & 70 & 20.7 & 19 & 23.8 & 30 & 23.6 & NS & 1.2 & NS & 1.5 & NS & 1.4 \\
\hline$D R 9 / X$ & 75 & 18.9 & 48 & 14.2 & 14 & 17.5 & 29 & 22.8 & NS & 0.71 & NS & 0.91 & NS & 1.3 \\
\hline$D R 8 / X$ & 10 & 2.5 & 6 & 1.8 & 1 & 1.3 & 7 & 5.5 & NS & 0.70 & NS & 0.49 & NS & 2.3 \\
\hline$D R X / X$ & 205 & 51.8 & 45 & 13.3 & 17 & 21.3 & 24 & 18.9 & $1.9 \times 10^{-28}$ & 0.14 & $5.4 \times 10^{-6}$ & 0.25 & $2.2 \times 10^{-10}$ & 0.2 \\
\hline
\end{tabular}

DR4: $D R B 1 * 0405-D Q B 1 * 0401$ haplotype; DR8: $D R B 1 * 0802-D Q B 1 * 0302$ haplotype; DR9: DRB1*0901-DQB1*0303 haplotype; X: haplotypes other than $D R 4, D R 8$ and $D R 9$

A, acute-onset; C, control participants; $\mathrm{F}$, fulminant; $p_{\mathrm{c}}, p$ values corrected for number of different haplotypes tested; SP, slowly progressive 
Table $3 D R B 1-D Q B 1$ genotypes in patients with acute-onset, fulminant and slowly progressive type 1 diabetes, and in control participants

\begin{tabular}{|c|c|c|c|c|c|c|c|c|c|c|c|c|c|c|}
\hline \multirow[t]{2}{*}{$D R B 1-D Q B 1$} & \multicolumn{2}{|c|}{ Control $(n=396)$} & \multicolumn{2}{|c|}{ Acute $(n=338)$} & \multicolumn{2}{|c|}{ Fulminant $(n=80)$} & \multicolumn{2}{|c|}{$\mathrm{SP}(n=127)$} & \multicolumn{2}{|l|}{ A vs $\mathrm{C}$} & \multicolumn{2}{|l|}{ F vs $\mathrm{C}$} & \multicolumn{2}{|l|}{$\mathrm{SP}$ vs $\mathrm{C}$} \\
\hline & $n$ & $\%$ & $n$ & $\%$ & $n$ & $\%$ & $n$ & $\%$ & $p_{\mathrm{c}}$ value & OR & $p_{\mathrm{c}}$ value & OR & $p_{\mathrm{c}}$ value & OR \\
\hline $\mathrm{S} / \mathrm{S}$ & 36 & 9.1 & 169 & 50.0 & 29 & 36.3 & 37 & 29.1 & $2.6 \times 10^{-35}$ & 10.0 & $4.7 \times 10^{-8}$ & 5.7 & $8.5 \times 10^{-8}$ & 4.1 \\
\hline $\mathrm{S} / \mathrm{N}$ & 109 & 27.5 & 109 & 32.2 & 27 & 33.8 & 61 & 48.0 & NS & 1.3 & NS & 1.3 & $1.1 \times 10^{-4}$ & 2.4 \\
\hline $\mathrm{S} / \mathrm{P}$ & 46 & 11.6 & 15 & 4.4 & 7 & 8.8 & 5 & 3.9 & $2.7 \times 10^{-3}$ & 0.35 & NS & 0.73 & NS & 0.31 \\
\hline $\mathrm{N} / \mathrm{N}$ & 119 & 30.1 & 34 & 10.1 & 6 & 7.5 & 18 & 14.2 & $8.3 \times 10^{-11}$ & 0.26 & $1.7 \times 10^{-4}$ & 0.19 & $2.4 \times 10^{-3}$ & 0.38 \\
\hline $\mathrm{N} / \mathrm{P}$ & 78 & 19.7 & 10 & 3.0 & 10 & 12.5 & 6 & 4.7 & $1.2 \times 10^{-12}$ & 0.12 & NS & 0.58 & $3.8 \times 10^{-4}$ & 0.20 \\
\hline $\mathrm{P} / \mathrm{P}$ & 8 & 2.0 & 1 & 0.3 & 1 & 1.3 & 0 & 0.0 & NS & 0.14 & NS & 0.61 & NS & - \\
\hline Non-S/non-S & 205 & 51.8 & 45 & 13.3 & 17 & 21.3 & 24 & 18.9 & $1.3 \times 10^{-28}$ & 0.14 & $3.6 \times 10^{-6}$ & 0.25 & $1.5 \times 10^{-10}$ & 0.22 \\
\hline $\mathrm{P} / \mathrm{X}$ & 132 & 33.3 & 26 & 7.7 & 18 & 22.5 & 11 & 8.7 & $2.0 \times 10^{-17}$ & 0.17 & NS & 0.58 & $3.4 \times 10^{-7}$ & 0.19 \\
\hline
\end{tabular}

A, acute-onset; C, control participants; F, fulminant; $\mathrm{P}$, protective haplotypes against acute-onset type 1 diabetes, $D R B 1^{*} 1501$ - $D Q B 1^{*} 0602$ and $D R B 1^{*} 1502-D Q B 1^{*} 0601 ; p_{\mathrm{c}}, p$ values corrected by multiplying by number of genotypes $(\times 6)$; $\mathrm{S}$, susceptible haplotypes for acute-onset type 1 diabetes, $D R B 1 * 0405-D Q B 1 * 0401, D R B 1 * 0802-D Q B 1 * 0302$ and $D R B 1 * 0901-D Q B 1 * 0303$; SP, slowly progressive; $\mathrm{X}$, any haplotype (e.g. $\mathrm{P} / \mathrm{X}=$ $\mathrm{P} / \mathrm{S}+\mathrm{P} / \mathrm{N}+\mathrm{P} / \mathrm{P})$

significantly higher in acute-onset (OR 2.1, $\left.p_{\mathrm{c}}=2.9 \times 10^{-3}\right)$ and slowly progressive diabetes $\left(\mathrm{OR} 2.6, p_{\mathrm{c}}=1.6 \times 10^{-2}\right)$ than in control participants, but this was not the case for fulminant type 1 diabetes (OR 1.2, NS) (ESM Table 5). In contrast, the frequency of $B^{*} 4002$ was significantly higher in fulminant (OR 2.9, $p_{\mathrm{c}}=0.017$ ), but not in acute-onset (OR 1.4, NS) and slowly progressive (OR 0.94, NS) type 1 diabetes, as compared with that in control participants (ESM Table 5). The frequency of $C^{*} 0803$ was significantly higher in fulminant (OR 9.6, $p_{\mathrm{c}}=0.022$ ), but not in acuteonset and slowly progressive type 1 diabetes, than in control participants (ESM Table 6); it was also significantly higher in fulminant than in acute-onset type 1 diabetes $\left(p_{\mathrm{c}}=\right.$ $0.03)$. The frequency of $C^{*} 0801$ was significantly higher in acute-onset, but not in fulminant and slowly progressive type 1 diabetes, than in control participants (ESM Table 6). The frequencies of $B^{*} 5201$ (ESM Table 5) and $C^{*} 1202$ (ESM Table 6) were significantly lower in acute-onset (OR $0.35, p_{\mathrm{c}}=5.1 \times 10^{-3}$; OR $0.37, p_{\mathrm{c}}=4.4 \times 10^{-3}$, respectively), but not in fulminant and slowly progressive type 1 diabetes, than in control participants.

Since most class I alleles showing association with type 1 diabetes were reported to be on haplotypes containing disease-susceptible class II HLA in the Japanese population [12], the participants were stratified by $D R B 1-D Q B 1$ to investigate whether or not these associations were secondary to linkage disequilibrium with $D R B 1-D Q B 1$ haplotypes. Class I alleles associated with acute-onset type 1 diabetes were in strong linkage disequilibrium with class II $D R B 1$ $D Q B 1$ haplotypes conferring susceptibility or resistance to acute-onset type 1 diabetes. The frequency of $B^{*} 5401$ was much higher in patients with $D R B 1 * 0405-D Q B 1 * 0401$ than in those without $\left(46.5 \%\right.$ vs $\left.15.9 \%, p=6.8 \times 10^{-7}\right)$, and the association of $B^{*} 5401$ with the disease was observed only in patients with $D R B 1^{*} 0405-D Q B 1^{*} 0401$ (acute-onset: OR $\left.4.0, p=3.7 \times 10^{-8}\right)$, but not in those without it (OR 0.86, NS) (ESM Table 7). Similarly, $B^{*} 4006$ and $C^{*} 0801$ were in linkage disequilibrium with $D R B 1 * 0901-D Q B 1 * 0303$ (37.3\% vs $12.0 \%, p=8.7 \times 10^{-6}$ and $40.2 \%$ vs $17.4 \%, p=$ $1.1 \times 10^{-4}$ in patients with and without $D R B 1 * 0901$ $D Q B 1^{*} 0303$, respectively); their association with the disease was observed in patients with, but not in patients without $D R B 1^{*} 0901-D Q B 1^{*} 0303$ (ESM Table 7). B*5201 and $C^{*} 1202$, which were negatively associated with the disease, were in linkage disequilibrium with $D R B 1 * 1502-D Q B 1 *$ $0601\left(40.0 \%\right.$ vs $5.2 \%, p=2.3 \times 10^{-4}$ and $43.7 \%$ vs $5.1 \%, p=$ $3.6 \times 10^{-5}$ in patients with and without $D R B 1 * 1502-D Q B 1 *$ 0601 , respectively). In contrast, the association of $B^{*} 4002$ with fulminant type 1 diabetes was observed regardless of the presence or absence of $D R B 1^{*} 0405-D Q B 1^{*} 0401$ and $D R B 1 * 0901-D Q B 1 * 0303$ (ESM Table 7).

Association with SNPs in HLA region As seen in Table 4 and ESM Fig. 1, a SNP located in class II $D Q B 1$ (rs1049107) was associated with all three subtypes, confirming that association with class II HLA is observed in all three subtypes of type 1 diabetes. A SNP located in TNF (rs1800610) was also associated with all three subtypes.

In addition to the peaks observed in all three subtypes, several peaks limited to one or two subtypes were observed. Several SNPs showed an association with fulminant, but not with acute-onset and slowly progressive type 1 diabetes. Among these were: (1) rs2071800, located in the coding region of DQA2; (2) rs2071552 and rs3763364 located in the $5^{\prime}$ untranslated region (UTR) and promoter region respectively of TAP2; and (3) rs2294689 located in the coding region of TTRAP. 
Table 4 Association of SNPs typed in the HLA region with acute, fulminant and slowly progressive type 1 diabetes

\begin{tabular}{|c|c|c|c|c|c|c|c|c|c|}
\hline \multirow[t]{2}{*}{ SNP ID } & \multirow{2}{*}{$\begin{array}{l}\text { dbSNP ID } \\
\text { (rs no.) }\end{array}$} & \multirow{2}{*}{$\begin{array}{l}\text { Gene } \\
\text { symbol }\end{array}$} & \multirow[t]{2}{*}{ Location } & \multicolumn{3}{|l|}{ OR $(95 \% \mathrm{CI})$} & \multicolumn{3}{|l|}{$p$ value } \\
\hline & & & & Acute & Fulminant & SP & Acute & Fulminant & SP \\
\hline 001 & 2294689 & TTRAP & CDS & $1.28(0.99-1.65)$ & $1.96(1.31-2.92)$ & $1.45(1.04-2.02)$ & NS & 0.0009 & 0.03 \\
\hline 002 & 2275906 & SLC17A4 & CDS & $1.00(0.59-1.71)$ & $0.65(0.25-1.73)$ & $0.79(0.38-1.67)$ & NS & NS & NS \\
\hline 003 & 1572982 & $H F E$ & Intron & $0.99(0.68-1.43)$ & $1.28(0.75-2.20)$ & $1.08(0.67-1.73)$ & NS & NS & NS \\
\hline 004 & 3736781 & $B T N 1 A 1$ & CDS & $1.12(0.85-1.48)$ & $0.66(0.41-1.05)$ & $0.98(0.68-1.40)$ & NS & NS & NS \\
\hline 005 & 3734576 & PRSS16 & 3'UTR & $0.76(0.42-1.38)$ & $0.75(0.28-2.01)$ & $1.00(0.48-2.07)$ & NS & NS & NS \\
\hline 006 & 2294481 & $D 6 S 2223$ & Intron & $1.12(0.87-1.44)$ & $1.05(0.71-1.56)$ & $1.23(0.88-1.71)$ & NS & NS & NS \\
\hline 007 & 1480646 & ZNF192 & Promoter & $1.03(0.73-1.45)$ & $1.23(0.74-2.05)$ & $0.91(0.58-1.45)$ & NS & NS & NS \\
\hline 008 & 2269553 & TRIM27 & Intron & $1.31(1.02-1.69)$ & $1.13(0.76-1.68)$ & $1.59(1.15-2.22)$ & 0.03 & NS & 0.006 \\
\hline 009 & 29230 & GABBRl & CDS & $0.74(0.54-1.02)$ & $0.72(0.43-1.19)$ & $0.99(0.67-1.46)$ & NS & NS & NS \\
\hline 010 & 2252711 & $M O G$ & Intron & $1.03(0.76-1.40)$ & $0.77(0.47-1.28)$ & $1.42(0.98-2.08)$ & NS & NS & NS \\
\hline 011 & 1736922 & $H L A-F$ & Intron & $1.46(1.13-1.88)$ & $1.15(0.77-1.72)$ & $0.90(0.64-1.27)$ & 0.004 & NS & NS \\
\hline 012 & 378971 & $H C G 9$ & CDS & $0.69(0.53-0.90)$ & $0.94(0.63-1.40)$ & $0.93(0.66-1.30)$ & 0.005 & NS & NS \\
\hline 013 & 2074479 & $R N F 39$ & CDS & $0.96(0.71-1.29)$ & $1.59(1.03-2.45)$ & $1.01(0.69-1.47)$ & NS & 0.04 & NS \\
\hline 014 & 2074474 & TRIM39 & CDS & $1.16(0.89-1.49)$ & $1.05(0.70-1.57)$ & $1.44(1.03-2.00)$ & NS & NS & 0.03 \\
\hline 015 & 3757388 & IRF5 & Promoter & $0.12(0.01-2.40)$ & $1.22(0.13-11.80)$ & $0.72(0.07-6.99)$ & NS & NS & NS \\
\hline 016 & 1265054 & C6orf15 & CDS & $0.69(0.53-0.90)$ & $0.79(0.53-1.19)$ & $0.74(0.52-1.03)$ & 0.005 & NS & NS \\
\hline 017 & 2073721 & TCF19 & CDS & $1.01(0.78-1.31)$ & $1.04(0.69-1.56)$ & $1.29(0.92-1.80)$ & NS & NS & NS \\
\hline 018 & 2523946 & $H C G 9$ & Promoter & $0.79(0.60-1.03)$ & $0.75(0.49-1.14)$ & $1.08(0.77-1.52)$ & NS & NS & NS \\
\hline 019 & 1049853 & $H L A-C$ & 3'UTR & $1.90(1.28-2.83)$ & $2.10(1.20-3.68)$ & $1.61(0.97-2.67)$ & 0.001 & 0.008 & NS \\
\hline 020 & $3819300^{\mathrm{a}}$ & $H L A-B$ & CDS & $0.56(0.38-0.82)$ & $0.64(0.35-1.17)$ & $0.95(0.61-1.50)$ & 0.003 & NS & NS \\
\hline 021 & 709052 & $H L A-B$ & CDS & $0.66(0.49-0.90)$ & $0.47(0.27-0.82)$ & $0.86(0.58-1.27)$ & 0.009 & 0.007 & NS \\
\hline 022 & 1050747 & $H L A-B$ & CDS & $0.73(0.51-1.04)$ & $1.10(0.65-1.86)$ & $0.59(0.35-0.97)$ & NS & NS & 0.04 \\
\hline 023 & 2534674 & $M I C B$ & Promoter & $1.38(1.06-1.79)$ & $1.05(0.70-1.58)$ & $1.42(1.02-1.98)$ & 0.02 & NS & 0.04 \\
\hline 024 & 2239527 & $B A T 1$ & $5^{\prime} \mathrm{UTR}$ & $0.77(0.59-1.01)$ & $0.78(0.51-1.18)$ & $0.85(0.60-1.20)$ & NS & NS & NS \\
\hline 025 & 2230365 & NFKBIL1 & CDS & $1.01(0.76-1.35)$ & $1.31(0.85-2.03)$ & $0.88(0.60-1.29)$ & NS & NS & NS \\
\hline 026 & 2239704 & $L T A$ & 5'UTR & $1.02(0.79-1.31)$ & $0.82(0.55-1.23)$ & $0.95(0.68-1.32)$ & NS & NS & NS \\
\hline 027 & 1800610 & $T N F$ & Intron & $2.87(2.16-3.82)$ & $1.95(1.27-2.99)$ & $2.17(1.51-3.11)$ & $1.0 \times 10^{-13 *}$ & 0.002 & $2.0 \times 10^{5 *}$ \\
\hline 028 & 2256974 & $L S T 1$ & Intron & $0.80(0.62-1.05)$ & $0.72(0.47-1.11)$ & $0.87(0.62-1.24)$ & NS & NS & NS \\
\hline 029 & 2736176 & $B A T 2$ & Promoter & $1.12(0.87-1.44)$ & $1.17(0.79-1.74)$ & $1.08(0.78-1.50)$ & NS & NS & NS \\
\hline 030 & 1046089 & $B A T 2$ & CDS & $1.31(1.00-1.71)$ & $1.38(0.92-2.08)$ & $1.05(0.74-1.50)$ & 0.047 & NS & NS \\
\hline 031 & 2242656 & BAT3 & Intron & $1.04(0.69-1.56)$ & $1.31(0.73-2.37)$ & $1.53(0.95-2.48)$ & NS & NS & NS \\
\hline 032 & 7992 & BAT4 & CDS & $1.36(1.05-1.76)$ & $1.34(0.89-2.00)$ & $1.29(0.92-1.80)$ & 0.02 & NS & NS \\
\hline 033 & 805282 & BAT5 & Intron & $1.32(1.02-1.71)$ & $1.28(0.86-1.91)$ & $1.32(0.95-1.85)$ & 0.04 & NS & NS \\
\hline 034 & 2075800 & HSPA1L & CDS & $0.96(0.74-1.24)$ & $0.91(0.61-1.35)$ & $0.92(0.66-1.29)$ & NS & NS & NS \\
\hline 035 & 7887 & EHMT2 & CDS & $0.69(0.52-0.91)$ & $0.56(0.35-0.89)$ & $0.93(0.65-1.32)$ & 0.01 & 0.01 & NS \\
\hline 036 & 2072634 & $C F B$ & CDS & $0.55(0.33-0.90)$ & $0.50(0.21-1.22)$ & $0.61(0.31-1.18)$ & 0.02 & NS & NS \\
\hline 037 & 3749966 & C6orf10 & CDS & $0.81(0.59-1.11)$ & $0.45(0.25-0.83)$ & $0.95(0.64-1.42)$ & NS & 0.009 & NS \\
\hline 038 & 2076530 & BTNL2 & CDS & $1.18(0.92-1.53)$ & $0.97(0.65-1.46)$ & $1.27(0.91-1.77)$ & NS & NS & NS \\
\hline 039 & 14004 & $H L A-D R A$ & $5^{\prime} \mathrm{UTR}$ & $1.36(1.04-1.77)$ & $1.12(0.74-1.69)$ & $1.28(0.91-1.80)$ & 0.02 & NS & NS \\
\hline 040 & 1049107 & $H L A-D Q B 1$ & CDS & $0.20(0.11-0.35)$ & $0.19(0.07-0.54)$ & $0.27(0.13-0.56)$ & $6.7 \times 10^{-10 *}$ & $5.6 \times 10^{-4} *$ & $1.7 \times 10^{-4} *$ \\
\hline 041 & 2071800 & $H L A-D Q A 2$ & CDS & $1.85(1.01-3.41)$ & $4.02(1.95-8.29)$ & $1.23(0.53-2.82)$ & 0.045 & $5.9 \times 10^{-5 *}$ & NS \\
\hline 042 & 3213484 & $H L A-D Q B 2$ & CDS & $0.83(0.62-1.10)$ & $0.58(0.35-0.95)$ & $0.91(0.63-1.32)$ & NS & 0.03 & NS \\
\hline 043 & 1049110 & $H L A-D Q B 2$ & CDS & $0.85(0.64-1.14)$ & $0.56(0.34-0.92)$ & $0.91(0.63-1.32)$ & NS & 0.02 & NS \\
\hline 044 & 2071554 & $H L A-D O B$ & CDS & $0.34(0.22-0.52)$ & $0.66(0.37-1.21)$ & $0.28(0.15-0.54)$ & $4.2 \times 10^{-6 *}$ & NS & $6.4 \times 10^{-5 *}$ \\
\hline 045 & 241441 & $T A P 2$ & CDS & $0.88(0.68-1.14)$ & $0.80(0.53-1.20)$ & $1.24(0.88-1.74)$ & NS & NS & NS \\
\hline 046 & 2071552 & $T A P 2$ & $5^{\prime} \mathrm{UTR}$ & $1.25(0.97-1.61)$ & $1.80(1.21-2.67)$ & $1.30(0.93-1.81)$ & NS & 0.004 & NS \\
\hline 047 & 3763364 & $T A P 2$ & Promoter & $1.31(1.00-1.71)$ & $2.19(1.46-3.28)$ & $1.21(0.86-1.71)$ & 0.048 & $1.2 \times 10^{-4 *}$ & NS \\
\hline
\end{tabular}


Table 4 (continued)

\begin{tabular}{|c|c|c|c|c|c|c|c|c|c|}
\hline \multirow[t]{2}{*}{ SNP ID } & \multirow{2}{*}{$\begin{array}{l}\text { dbSNP ID } \\
\text { (rs no.) }\end{array}$} & \multirow{2}{*}{$\begin{array}{l}\text { Gene } \\
\text { symbol }\end{array}$} & \multirow[t]{2}{*}{ Location } & \multicolumn{3}{|l|}{ OR $(95 \% \mathrm{CI})$} & \multicolumn{3}{|l|}{$p$ value } \\
\hline & & & & Acute & Fulminant & SP & Acute & Fulminant & SP \\
\hline 048 & 2071543 & PSMB8 & CDS & $0.70(0.48-1.03)$ & $1.32(0.78-2.23)$ & $0.64(0.38-1.08)$ & NS & NS & NS \\
\hline 049 & 2071463 & PSMB8 & $5^{\prime} \mathrm{UTR}$ & $1.10(0.85-1.43)$ & $0.85(0.56-1.29)$ & $1.03(0.73-1.45)$ & NS & NS & NS \\
\hline 050 & $1800453^{b}$ & $T A P 1$ & CDS & $0.94(0.62-1.41)$ & $0.48(0.21-1.08)$ & $1.19(0.72-1.97)$ & NS & NS & NS \\
\hline 051 & 2071536 & $T A P 1$ & CDS & $0.31(0.18-0.56)$ & $0.64(0.29-1.39)$ & $0.37(0.17-0.80)$ & $3.4 \times 10^{-5 *}$ & NS & 0.009 \\
\hline 052 & 17587 & PSMB9 & CDS & $1.37(1.02-1.84)$ & $1.68(1.08-2.60)$ & $1.42(0.97-2.07)$ & 0.04 & 0.02 & NS \\
\hline 053 & 1042337 & $H L A-D M B$ & CDS & $1.05(0.80-1.38)$ & $1.04(0.68-1.58)$ & $1.08(0.76-1.52)$ & NS & NS & NS \\
\hline 054 & 150359 & $H L A-D M A$ & Promoter & $1.32(1.02-1.70)$ & $1.02(0.68-1.51)$ & $1.46(1.05-2.03)$ & 0.03 & NS & 0.03 \\
\hline 055 & 516535 & $B R D 2$ & CDS & $1.30(1.01-1.68)$ & $1.01(0.68-1.49)$ & $1.44(1.04-2.00)$ & 0.04 & NS & 0.03 \\
\hline 056 & 375256 & $H L A-D O A$ & CDS & $1.16(0.88-1.55)$ & $1.02(0.65-1.60)$ & $0.88(0.59-1.29)$ & NS & NS & NS \\
\hline 057 & 3097671 & $H L A-D P B 1$ & Intron & $0.56(0.38-0.83)$ & $0.68(0.37-1.25)$ & $0.48(0.28-0.84)$ & 0.003 & NS & 0.009 \\
\hline 058 & 1799908 & COL11A2 & CDS & $0.87(0.64-1.17)$ & $0.61(0.36-1.02)$ & $0.87(0.59-1.29)$ & NS & NS & NS \\
\hline 059 & 2072915 & $R X R B$ & 3'UTR & $0.88(0.65-1.20)$ & $0.67(0.40-1.12)$ & $0.87(0.58-1.30)$ & NS & NS & NS \\
\hline 060 & 383711 & $H S D 17 B 8$ & Intron & $0.54(0.42-0.70)$ & $0.64(0.43-0.96)$ & $0.59(0.42-0.83)$ & $2.7 \times 10^{-6 *}$ & 0.03 & 0.002 \\
\hline 061 & 213208 & RING1 & Intron & $0.68(0.52-0.89)$ & $0.81(0.53-1.24)$ & $0.64(0.44-0.92)$ & 0.005 & NS & 0.02 \\
\hline 062 & 213199 & VPS52 & CDS & $0.67(0.51-0.89)$ & $1.02(0.67-1.55)$ & $0.54(0.36-0.80)$ & 0.005 & NS & 0.002 \\
\hline 063 & $466384^{\mathrm{c}}$ & WDR46 & CDS & $0.94(0.54-1.62)$ & $0.40(0.12-1.36)$ & $0.65(0.29-1.47)$ & NS & NS & NS \\
\hline 064 & 456261 & PFDN6 & Intron & $0.66(0.50-0.88)$ & $0.95(0.62-1.46)$ & $0.57(0.39-0.85)$ & 0.004 & NS & 0.005 \\
\hline 065 & 1059288 & $T A P B P$ & 3'UTR & $0.66(0.50-0.87)$ & $0.97(0.64-1.47)$ & $0.57(0.39-0.83)$ & 0.003 & NS & 0.003 \\
\hline 066 & 2071888 & TAPBP & CDS & $0.65(0.49-0.85)$ & $0.93(0.61-1.41)$ & $0.57(0.40-0.83)$ & 0.002 & NS & 0.003 \\
\hline 067 & 2073525 & $D A X X$ & Promoter & $0.65(0.49-0.85)$ & $0.91(0.60-1.37)$ & $0.59(0.41-0.86)$ & 0.002 & NS & 0.005 \\
\hline 068 & 2274730 & ZВТВ9 & 5'UTR & $0.60(0.45-0.81)$ & $0.94(0.60-1.48)$ & $0.72(0.50-1.06)$ & $6.5 \times 10^{-4} *$ & NS & NS \\
\hline
\end{tabular}

${ }^{\mathrm{a}}$ Now merged into rs $2308655 ;{ }^{\mathrm{b}}$ now merged into rs1135216; ${ }^{\mathrm{c}}$ now merged into rs14398

${ }^{*} p$ values that remained significant after correction for number of SNPs genotyped $(\times 68)$

In contrast, SNPs located in the coding regions of $D O B$ (rs2071554) and TAP1 (rs2071536) were associated with acute-onset and slowly progressive, but not with fulminant type 1 diabetes. Similarly, an association was suggested between several SNPs located in the most centromeric region, e.g. rs3097671 in $D P B 1$ and rs383711 in $H S D 17 B 8$, and both acute-onset and slowly progressive, but not fulminant type 1 diabetes. SNPs located in the region centromeric to DPB1 (rs213208, rs213199, rs45261, rs1059288, rs2071888 and rs2073525) also showed a tendency for association with acute-onset and slowly progressive, but not fulminant type 1 diabetes.

To investigate whether or not these associations were secondary to linkage disequilibrium with susceptible and protective $D R B 1-D Q B 1$ haplotypes, the participants were stratified by $D R B 1-D Q B 1$. A minor $\mathrm{T}$ allele of $D Q A 2$, which was strongly associated with fulminant type 1 diabetes (OR 4.02, $p=6 \times 10^{-5}$ ), was in strong linkage disequilibrium with $D R B 1 * 0405-D Q B 1 * 0401$ in control participants, with $19.1 \%$ frequency in participants with, as compared with $2.8 \%$ frequency in participants without $D R B 1^{*} 0405$ $D Q B 1 * 0401\left(p=4.0 \times 10^{-5}\right)$. The association of $D Q A 2 * T$ with the disease was observed only in patients with $D R B 1^{*}$ $0405-D Q B 1^{*} 0401$ (OR 10.3, $p=1.6 \times 10^{-8}$ ), but not in patients without $D R B 1 * 0405-D Q B 1 * 0401$ (OR 0.63 , NS).

In contrast, SNPs whose minor alleles showed a negative association with acute-onset and slowly progressive type 1 diabetes were in linkage disequilibrium with protective $D R B 1-D Q B 1$ haplotypes, i.e. either $D R B 1 * 1501$ (rs2071536 in TAP1, $p=1.8 \times 10^{-14}$ ) or DRB1*1502 (rs383711: $p=5.6 \times$ $10^{-5}$, rs45261: $p=2.4 \times 10^{-6}$, rs1059288: $p=4.0 \times 10^{-6}$, rs2071888: $p=3.5 \times 10^{-6}$, rs2073525: $p=7.8 \times 10^{-6}$ ) or both (rs2071554 in $D O B, p=1.8 \times 10^{-10}$ for $D R B 1^{*} 1501$ and $p=$ $2.3 \times 10^{-21}$ for $D R B 1^{*} 1502$ ).

\section{Discussion}

The present study demonstrates that class II HLA is associated with all three subtypes of type 1 diabetes, but the alleles, haplotypes and genotypes associated with the disease are markedly different among the three subtypes. Basically, the alleles and haplotypes associated with acute- 
onset and slowly progressive type 1 diabetes were similar, whereas those associated with fulminant type 1 diabetes were mostly different from those in the other two subtypes of type 1 diabetes, as shown by the lack, in fulminant type 1 diabetes, of (1) protection conferred by $D R B 1^{*} 1501$ $D Q B 1^{*} 0602$, a highly protective haplotype against acuteonset type 1 diabetes, and (2) susceptibility conferred by the $D R B 1^{*} 0802-D Q B 1^{*} 0302$ and $D R 4 / 8$ genotype, which confers strong susceptibility to acute-onset type 1 diabetes.

$D R B 1 * 0405-D Q B 1^{*} 0401$ was associated with fulminant as well as acute-onset and slowly progressive type 1 diabetes, but the magnitude of the effect differed, particularly in the homozygous form, playing a key role in fulminant type 1 diabetes. The fact that neither $D R B 1^{*}$ 1501-DQB1*0602 nor $D Q B 1 * 0302$ affected susceptibility and only Asian-specific $D R B 1^{*} 0405-D Q B 1^{*} 0401$ conferred susceptibility in fulminant type 1 diabetes may explain the marked difference in incidence of fulminant type 1 diabetes among different ethnic groups. Fulminant type 1 diabetes has been reported in Asian populations, comprising up to $20 \%$ of adult-onset type 1 diabetes in Japan [2] and $7 \%$ of Korean type 1 diabetes [3]; in these populations $D R B 1 * 0405-D Q B 1 * 0401$ is a common haplotype. In contrast, fulminant type 1 diabetes appears to be extremely rare in white populations $[2,13]$, in whom the $D R B 1^{*} 0405-D Q B 1 * 0401$ haplotype is also very rare. This may also reflect the difference in aetiology between fulminant and other subtypes of type 1 diabetes, i.e. autoimmune aetiology in acute-onset and slowly progressive type 1 diabetes, and idiopathic aetiology in fulminant type 1 diabetes $[1,2]$. Several lines of evidence suggest viral infection in genetically susceptible individuals as a cause of fulminant type 1 diabetes [2, 14-16]. DRBI*0405$D Q B 1^{*} 0401$ was reported to be associated with immunological responses against certain viruses [17].

Slowly progressive type 1 diabetes is similar, but not identical to latent autoimmune diabetes in adults (LADA) in white populations [18] and is defined by autoimmune aetiology as reflected by positivity for islet-related autoantibodies, but slower progression to an insulin-dependent stage than in acute-onset type 1 diabetes [4]. LADA is defined by positivity for islet-related autoantibodies as in the case of slowly progressive type 1 diabetes, but progression to an insulin-dependent stage is not a necessary part of its definition [18]. Slowly progressive type 1 diabetes is more likely to be a mild form of acute-onset type 1 diabetes [4]. Consistent with this, the class II alleles and haplotypes associated with slowly progressive type 1 diabetes in the present study were similar to those in acuteonset type 1 diabetes. The similarity of HLA alleles and haplotypes between acute-onset and slowly progressive type 1 diabetes suggests that the phenotypic difference between these two subtypes of type 1 diabetes may not be due to differences in alleles and haplotypes of class II HLA. One possibility is a difference in presence of susceptible haplotypes between slowly progressive and acute-onset type 1 diabetes. As shown in Table 3, the presence of both two $(\mathrm{S} / \mathrm{S})$ and of one $(\mathrm{S} / \mathrm{N})$ susceptible haplotypes conferred susceptibility to slowly progressive type 1 diabetes, whereas for acute-onset type 1 diabetes only the former $(\mathrm{S} / \mathrm{S})$, but not the latter $(\mathrm{S} / \mathrm{N})$ conferred susceptibility, suggesting that differences in numbers of susceptible haplotypes affect the speed of disease progression, leading to the difference between acute-onset and slowly progressive forms of type 1 diabetes.

In addition to class II HLA, several loci, including class I HLA, showed some evidence of association with type 1 diabetes. Most alleles of class I HLA that were associated with acute-onset and slowly progressive type 1 diabetes (ESM Tables 4, 5, and 6) appeared to be secondary to linkage disequilibrium between these alleles and diseaserelated class II alleles, e.g. $B^{*} 5401$ with $D R B 1 * 0405-D Q B 1^{*}$ $0401, B^{*} 4006$ and $C^{*} 0801$ with $D R B 1^{*} 0901-D Q B 1^{*} 0303$, and $B^{*} 5201$ and $C^{*} 1202$ with $D R B 1^{*} 01502-D Q B 1^{*} 0601$. However, the class I alleles associated with fulminant type 1 diabetes were different from those associated with acuteonset type 1 diabetes in that $B^{*} 5401$, which was increased in acute-onset and slowly progressive type 1 diabetes, was not increased and instead $B^{*} 4002$ was increased. $B^{*} 4002$ was reported to be on haplotypes containing $D R B 1^{*} 0405-D Q B 1^{*}$ 0401 and $D R B 1^{*} 0901-D Q B 1^{*} 0303$ in the Japanese population, although the haplotype frequencies are very low [12]. However, association of $B^{*} 4002$ with fulminant type 1 diabetes cannot be explained by linkage disequilibrium with these class II haplotypes, because the association was observed regardless of the presence or absence of the $D R B 1^{*}$ 0405-DQB1*0401 and DRB1*0901-DQB1*0303 haplotypes (ESM Table 7), suggesting that genes outside class II HLA, including class I HLA, contribute to the difference between fulminant and other subtypes of type 1 diabetes.

To further clarify the contribution of genes outside class II HLA to the phenotypic difference between the three subtypes of type 1 diabetes, we genotyped SNPs in an $8.5 \mathrm{Mb}$ region of the extended HLA that ranged from a locus $1.5 \mathrm{Mb}$ telomeric to $H F E$ to a locus $0.5 \mathrm{Mb}$ centromeric to $D P B 1$. In addition to the association observed in all three subtypes, an association limited to one or two subtypes was also observed, which may contribute to the phenotypic differences between the three disease subtypes. Among these were SNPs in TAP2 and DQA2, which were associated with fulminant, but not with acute-onset or slowly progressive type 1 diabetes, with minor alleles conferring disease susceptibility. In contrast, a SNP in $D O B$ was associated with acute-onset and slowly progressive, but not with fulminant, type 1 diabetes, with the minor allele being protective against the disease, as previously reported 
for acute-onset type 1 diabetes in a white population [19]. A similar tendency of association was also suggested for several SNPs located in the most centromeric region (rs45261, rs1059288, rs2071888, rs2073525) (Table 4).

Although extensive effort has been put into collecting samples, particularly for fulminant type 1 diabetes, the number of samples in the present study is still modest for this kind of study. Further studies with dense SNPs and a larger number of participants are necessary to clarify whether or not the association of these SNPs with each subtype is real and independent of other nearby genes. In addition, the contribution of genes outside the extended HLA, as well as of those on different chromosomes to the disease, should also be studied in these three subtypes of type 1 diabetes.

In conclusion, the present study demonstrates that class II HLA is associated with three subtypes of type 1 diabetes, fulminant, acute-onset and slowly progressive forms, but the alleles, haplotypes and genotypes associated with the disease differ among the three subtypes. The association with HLA in fulminant type 1 diabetes is qualitatively different from that in other subtypes of type 1 diabetes, which may reflect the difference in aetiology between fulminant and other subtypes of type 1 diabetes. In contrast, the association with HLA in slowly progressive type 1 diabetes is qualitatively similar to, but quantitatively different from that in acute-onset type 1 diabetes. Given that in this study a substantial number of patients with fulminant type 1 diabetes or with well characterised slowly progressive type 1 diabetes were recruited only in Japan, further studies with dense genetic markers, including whole-genome association studies comparing these three subtypes of type 1 diabetes, are necessary. Such studies are now underway.

Acknowledgements We thank M. Yamaoka-Sageshima, Y. Ishibashi and Y. Ogasawara for their technical assistance. This work was partly supported by a grant from the Japan Diabetes Society, a Grant-in-Aid for Scientific Research on Priority Areas (C) (Medical Genome Science [Millennium Genome Project] and Comprehensive Genomics) and a Grant-in-Aid for Scientific Research from the Ministry of Education, Culture, Sports, Science and Technology of Japan.

Duality of interest The authors declare that there is no duality of interest associated with this manuscript.

\section{References}

1. Imagawa A, Hanafusa T, Miyagawa J, Matsuzawa Y (2000) A novel subtype of type 1 diabetes mellitus characterized by a rapid onset and an absence of diabetes-related antibodies. N Engl J Med 342:301-307
2. Imagawa A, Hanafusa T (2007) Fulminant type 1 diabetes: a novel clinical entity requiring special attention by all medical practitioners. Nat Clin Prac Endocrinol Metab 3:36-45

3. Cho YM, Kim JT, Ko KS et al (2007) Fulminant type 1 diabetes in Korea: high prevalence among patients with adult-onset type 1 diabetes. Diabetologia 50:2276-2279

4. Kobayashi T, Tamemoto K, Nakanishi K et al (1993) Immunogenetic and clinical characterization of slowly progressive IDDM. Diabetes Care 16:780-788

5. Todd J, Bell JI, MacDevitt HO (1987) HLA-DQ beta gene contributes to susceptibility and resistance to insulin-dependent diabetes mellitus. Nature 329:599-604

6. Imagawa A, Hanafusa T, Uchigata $Y$ et al (2005) Different contribution of class II HLA in fulminant and typical autoimmune type 1 diabetes mellitus. Diabetologia 48:294-300

7. Bannai M, Higuchi K, Akesaka T et al (2004) Single-nucleotidepolymorphism genotyping for whole-genome-amplified samples using automated fluorescence correlation spectroscopy. Anal Biochem 327:215-221

8. Ikegami H, Kawaguchi Y, Yamato E et al (1992) Analysis by the polymerase chain reaction of histocompatibility leucocyte antigenDR9-linked susceptibility to insulin-dependent diabetes mellitus. J Clin Endocrinol Metab 75:1381-1385

9. Awata T, Kuzuya T, Matsuda A et al (1992) Genetic analysis of HLA class II alleles and susceptibility to type 1 (insulin-dependent) diabetes mellitus in Japanese subjects. Diabetologia 35:419-424

10. Kawabata Y, Ikegami H, Kawaguchi Y et al (2002) Asian-specific HLA haplotypes reveal heterogeneity of the contribution of HLADR and -DQ haplotypes to susceptibility to type 1 diabetes. Diabetes 51:545-551

11. Murao S, Makino H, Kaino $Y$ et al (2004) Differences in the contribution of HLA-DR and -DQ haplotypes to susceptibility to adult- and childhood-onset type 1 diabetes in Japanese patients. Diabetes 53:2684-2690

12. Saito S, Ota S, Yamada E, Inoko H, Ota M (2000) Allele frequencies and haplotypic associations defined by allelic DNA typing at HLA class I and class II loci in the Japanese population. Tissue Antigens 56:522-529

13. Moreau C, Drui D, Arnault-Ouary G, Charnonnel B, Chaillous L, Cariou B (2008) Fulminant type 1 diabetes in Caucasians: a report of three cases. Diabetes Metab 34:529-532

14. Imagawa A, Hanafusa T (2006) Pathogenesis of fulminant type 1 diabetes. Rev Diabet Stud 3:169-177

15. Imagawa A, Hanafusa T, Makino H, Miyagawa JI, Juto P (2005) High titres of IgA antibodies to enterovirus in fulminant type 1 diabetes. Diabetologia 48:290-293

16. Shimada A, Maruyama T (2004) Encephalomyocarditis-virusinduced diabetes model resembles "fulminant" type 1 diabetes in humans. Diabetologia 47:1854-1855

17. Mineta M, Tanimura M, Tana T, Yssel H, Kashiwagi S, Sasazuki T (1996) Contribution of HLA class I and class II alleles to the regulation of antibody production to hepatitis B surface antigen in humans. Int Immunol 8:525-531

18. Zimmet PZ, Tuomi T, Mackay IR et al (1994) Latent autoimmune diabetes mellitus in adults (LADA): the role of antibodies to glutamic acid decarboxylase in diagnosis and prediction of insulin dependency. Diabet Med 11:299-303

19. Zavattari P, Lampis R, Motzo C et al (2001) Conditional linkage disequilibrium analysis of a complex disease superlocus, IDDMI in the HLA region, reveals the presence of independent modifying gene effects influencing the type 1 disease risk encoded by the major HLA-DQB1, -DRB1 disease loci. Hum Mol Genet 10:881-889 